\title{
A triangular coastal element developed for use in finite difference tidal models
}

\author{
David McInerney* John Noye ${ }^{\dagger}$
}

(Received 7 August 2000)

\begin{abstract}
In numerical models of environmental flows it is often necessary to implement impermeable boundaries of complicated shape. For example, when modelling the spread of pollutants in streams, the dispersion
\end{abstract}

* Department of Applied Mathematics, University of Adelaide, Adelaide, SA 5005, Australia.

$\dagger$ National Tidal Facility, Flinders University of South Australia, Adelaide, SA 5001, Australia; seconded from the Department of Applied Mathematics, University of Adelaide. mailto: jnoye@maths.adelaide.edu.au

${ }^{0}$ See http://anziamj . austms . org. au/V42/CTAC99/Mcin for this article and ancillary services, (C) Austral. Mathematical Soc. 2000. Published 27 Nov 2000. 
of contaminants in lakes and estuaries or the final coastal destination of an oil spill, the land-water boundary is not simply defined. Currently such boundaries are best represented using Finite Element (FE) techniques. However FE techniques are both computationally expensive and difficult to implement. As a result Finite Difference Methods (FDMs) on rectangular grids have traditionally been used to model environmental flows.

In this paper a triangular boundary element for finite difference models of tidal flows in coastal regions, which improves boundary resolution while maintaining computational efficiency, is developed and tested. Numerical predictions using the new approach are compared with predictions obtained using the traditional stepped boundary and an analytic solution for depth-integrated flow in an idealised estuary.

\section{Contents}

1 Introduction

C938

2 Mathematical formulation

C942

3 Numerical procedure

C943

4 Incorporating triangular coastal elements

C945

5 Testing the model

C948 
6 Conclusion

C951

References

C952

\section{Introduction}

In standard finite difference tidal models, such as those developed in [2]-[5], the region of interest is covered by a grid leading to a set of grid elements consisting of two types, land and water elements. The actual coastline must then be approximated as the boundary between regions containing land and regions containing water. An example of such a grid is shown in Figure 1. This model approximation of northern Spencer Gulf appears to reasonably represent the actual coastline. Certainly, it allows recognition of the region of interest. However, modelling the coast in such a manner does have drawbacks.

The discretized coastline can sometimes be a poor approximation to the real thing. In particular, stretches of coast which run at an angle of approximately $45^{\circ}$ to the grid are poorly represented. The coastline in the south-east corner of the northern Spencer Gulf region is an example of this. It contains a number of $90^{\circ}$ corners where modelled results, particularly velocities, will be noticeably affected. While currents are expected to run parallel to the coast, velocities will zig-zag in an attempt to follow the modelled coastline. 


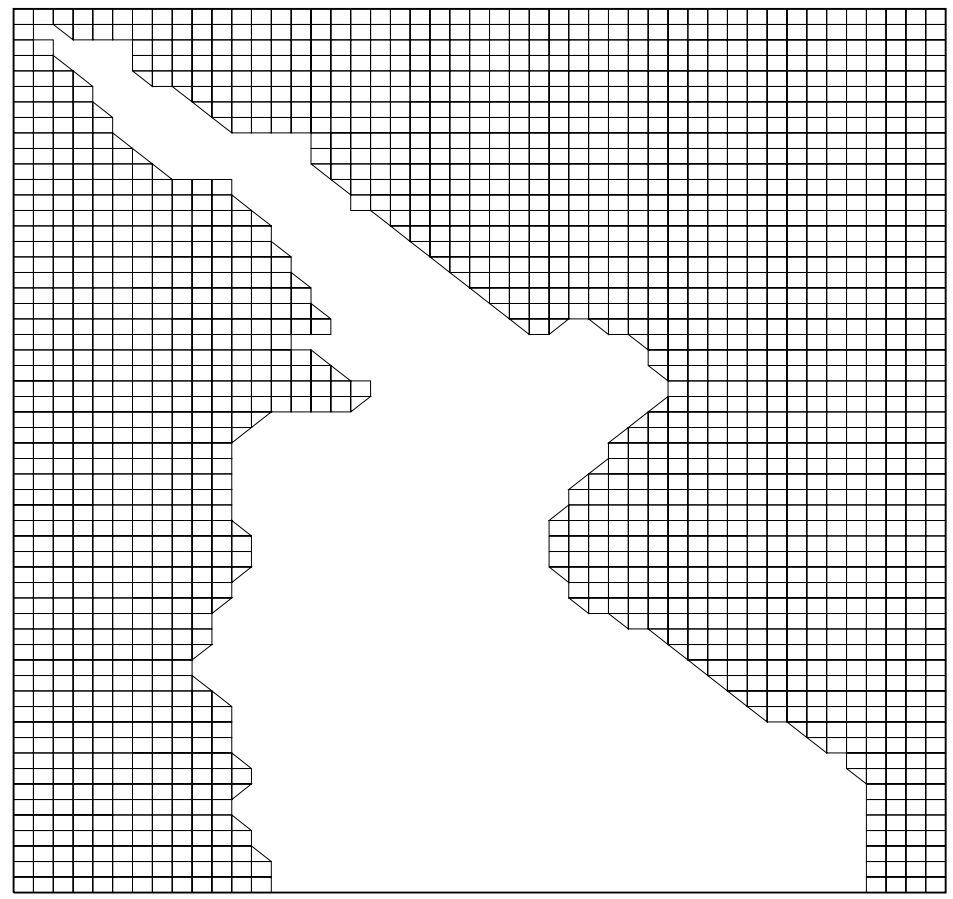

Figure 1: A standard finite difference computational boundary for the northern Spencer Gulf region. 
One application which relies heavily on the accuracy of the coastline is oil spill tracking. If the modelled coastline consists of a large number of $90^{\circ}$ corners, the oil may end up trapped in the artificial bays. A more realistic smooth coastline would allow the oil to move along the boundaries.

The ideal method of increasing the accuracy of the coastline would require minimal increases in computer resources. This would suggest a method which is only employed around coastal areas, but at the same time it must not complicate calculations. The method introduced in this paper allows the addition of four new coastal elements to complement the land and water elements used in standard models. These elements will contain half land and half water, with the coast running diagonally from one corner of the grid box to the opposite corner.

Figure 2 shows the grid representation of the northern Spencer Gulf region when these triangular elements are used. The model coastline is a much better approximation of the actual coast, particularly those stretches of coast which run at a sharp angle to the grid. However, for these elements to be used, a technique for incorporating them in the same manner as sea elements must be developed. 


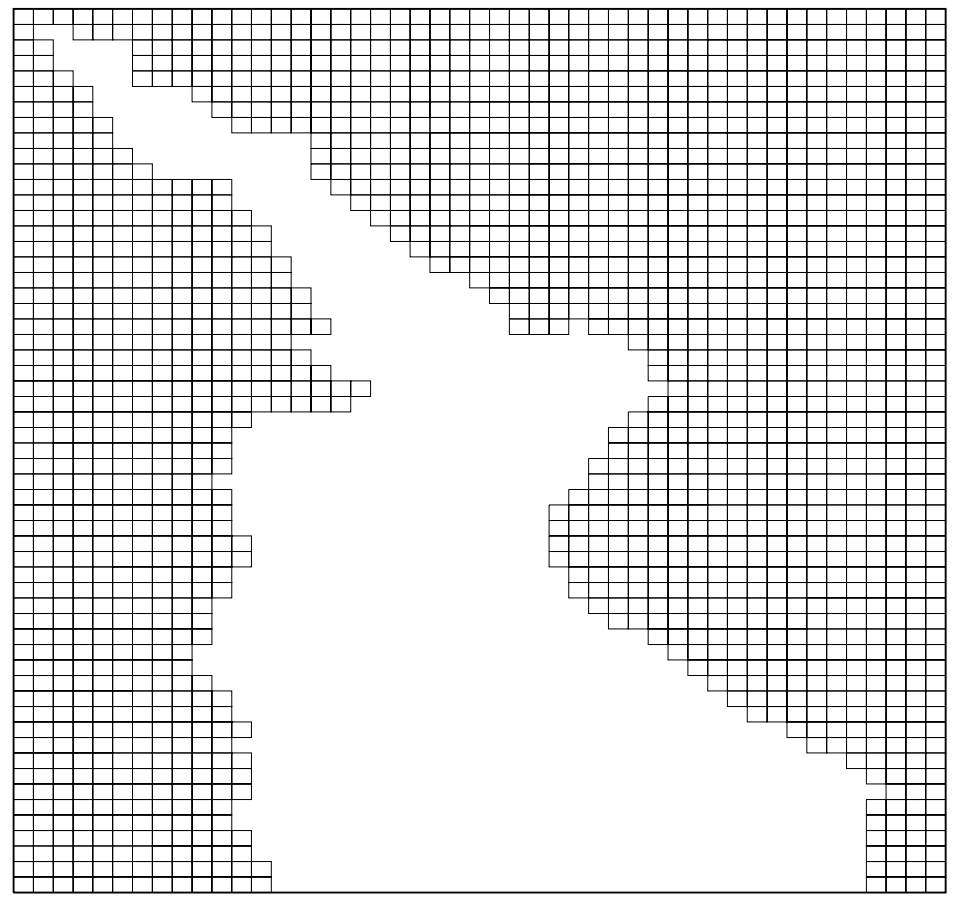

FIGURE 2: The triangulated computational boundary for the northern Spencer Gulf region. 


\section{Mathematical formulation}

The test case used to illustrate the effectiveness of the triangular elements considers tidal oscillations in a semi-infinite rectangular constant depth bay. The 2-D linearized depth-integrated equations of mass and momentum conservation, namely,

$$
\begin{aligned}
\frac{\partial \zeta}{\partial t} & =-\frac{\partial U}{\partial x}-\frac{\partial V}{\partial y}, \\
\frac{\partial U}{\partial t} & =-g h \frac{\partial \zeta}{\partial x}-C U+f V, \\
\frac{\partial V}{\partial t} & =-g h \frac{\partial \zeta}{\partial y}-C V-f U,
\end{aligned}
$$

are used to describe the behaviour of the surface elevation and currents. Here $t$ is time in seconds $(\mathrm{s}), x$ and $y$ are the cartesian spatial coordinates in the plane of mean sea level (MSL), measured in meters $(\mathrm{m}), h$ is the depth of the sea bed below MSL (a constant depth of $20 \mathrm{~m}$ is considered), $\zeta$ is the surface elevation (m) above MSL, $U$ and $V$ are the depth-integrated components of fluid velocity $\left(\mathrm{m}^{2} \mathrm{~s}^{-1}\right)$ in the $x$ and $y$ directions, $f$ is the Coriolis parameter, taken as $-8.37 \times 10^{-5} \mathrm{~s}^{-1}$ (corresponding to a latitude of $35^{\circ}$ South), $g$ is the gravitational acceleration, $9.81 \mathrm{~ms}^{-2}$, and $C$ is the coefficient of linear friction, a constant $0.00025 \mathrm{~s}^{-1}$ over the entire bay.

The fluid starts from rest, that is $\zeta=U=V=0$ at time $t=0$, and velocities perpendicular to the coastal boundary are zero for time $t>0$. 
Finally all elevations on or outside the open boundary are specified at each time level.

\section{$3 \quad$ Numerical procedure}

Equations (1-3) are approximately solved using FDMs with computations performed on an Arakawa $\mathrm{C}$ grid (see [1]). Figure 3 shows the $(j, k)$-th gridbox with the variables $\zeta, U$ and $V$ defined at the indicated positions. The depths $h$ are specified at the $\zeta$ points and $f$ and $C$ are considered constant.

The relationships between the scripted variables and their continuous counterparts are

$$
\begin{aligned}
h_{j, k} & =h(j \Delta x, k \Delta y), \\
\zeta_{j, k}^{n} & =\zeta(j \Delta x, k \Delta y, n \Delta t), \\
U_{j, k}^{n} & =U((j+1 / 2) \Delta x, k \Delta y, n \Delta t), \\
V_{j, k}^{n} & =V(j \Delta x,(k+1 / 2) \Delta y, n \Delta t) .
\end{aligned}
$$

The formulae used to numerically integrate (1-3) from time level $n$ to $n+1$ at the $\zeta, U$ and $V$ points inside the bay are:

$$
\begin{aligned}
\zeta_{j, k}^{n+1} & =\zeta_{j, k}^{n}-r_{x}\left(U_{j, k}^{n}-U_{j-1, k}^{n}\right)-r_{y}\left(V_{j, k}^{n}-V_{j, k-1}^{n}\right), \\
U_{j, k}^{n+1} & =U_{j, k}^{n}-g h r_{x}\left(\zeta_{j+1, k}^{n+1}-\zeta_{j, k}^{n+1}\right)-C \Delta t U_{j, k}^{n}
\end{aligned}
$$




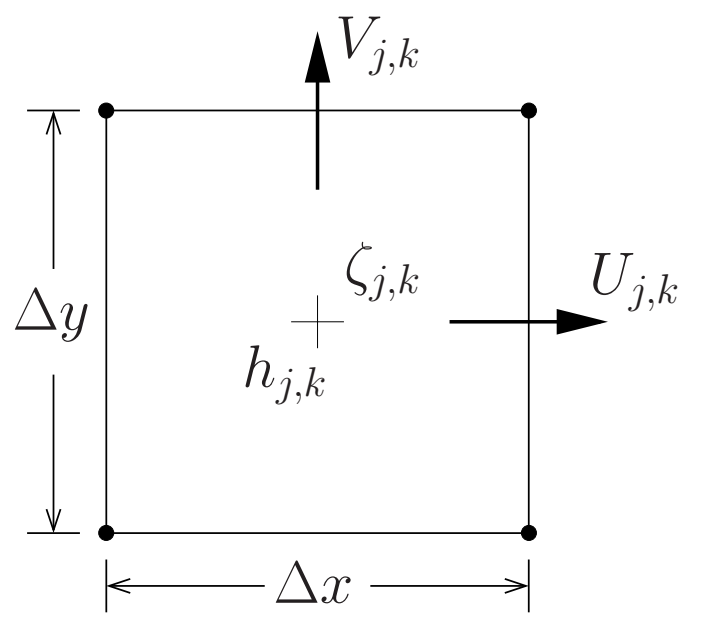

Figure 3: Position and variables within the $(j, k)$-th grid box. 


$$
\begin{gathered}
+f \Delta t\left(V_{j, k-1}^{n}+V_{j+1, k-1}^{n}+V_{j, k}^{n}+V_{j+1, k}^{n}\right) / 4, \\
V_{j, k}^{n+1}=\quad V_{j, k}^{n}-g h r_{y}\left(\zeta_{j, k+1}^{n+1}-\zeta_{j, k}^{n+1}\right)-C \Delta t V_{j, k}^{n} \\
\quad-f \Delta t\left(U_{j-1, k}^{n}+U_{j, k}^{n}+U_{j-1, k+1}^{n}+U_{j, k+1}^{n}\right) / 4,
\end{gathered}
$$

where $r_{x}=\Delta t / \Delta x$ and $r_{y}=\Delta t / \Delta y$. This system of equations has been shown to be von Neumann stable provided

$$
\Delta t<\min \left\{\frac{-C+\sqrt{C^{2}+16 g h A}}{4 g h A}, \frac{-D+\sqrt{D^{2}+8 g h A}}{2 g h A}, \frac{1}{D}\right\},
$$

where $A=(\Delta x)^{-2}+(\Delta y)^{-2}$ and $D=\left(C^{2}+f^{2}\right) / C$ (see [6]).

\section{Incorporating triangular coastal elements}

The method used to incorporate triangular elements into the numerical model is now described. The standard elements used in finite difference tidal models are those made up wholly of land and wholly of water. These elements will still make up the majority of the grid, but four new triangular elements, with the coast running from one corner to the opposite corner, will be introduced.

Allocation of element types to grid boxes is performed using a coastline fitting subroutine in the program. Using bathymetric data and present sea level as the sole inputs, the coastline fitter considers which of the four types of triangular elements would exist in every grid box before determining whether 
the element is land, water or triangular, from the present sea level. This automated process replaces the manual allocation of elements traditionally performed by eye and removes much of the manual labour involved in the usual initialisation of the model.

The process by which the triangular land elements are handled within the numerical model must meet several requirements. For example, the equations of fluid motion must be satisfied in a manner consistent with the layout of the triangular elements. The ideal technique would be one that satisfies the physical properties expected of a triangular land element, and preferably makes use of the same finite difference formulae being used for the open water elements.

One physical property which must be satisfied is the impermeability of the coastal boundary running through the centre of each triangular element. This requires that there be no flow perpendicular to the boundary, which means the flow velocity at the centre of each triangular element must be tangential to this boundary. A simple reflection about the diagonal boundary is used to satisfy this property. This is illustrated in Figure 4 for a north-east triangular land element with sides $\Delta x=\Delta y$.

The inflow through the southern side of the grid box $\left(V_{\text {south }}\right)$ is reflected about the coastline to give an equal inflow $\left(V_{\text {south }}\right)$ through the eastern side. Similarly an inflow of $U_{\text {west }}$ is forced through the northern side of the grid box. Thus if the $(j, k)$-th grid box contains a north-east triangular element the formulae $U_{j, k}^{n}=-V_{j, k-1}^{n}$ and $V_{j, k}^{n}=-U_{j-1, k}^{n}$ would be applied at the end 


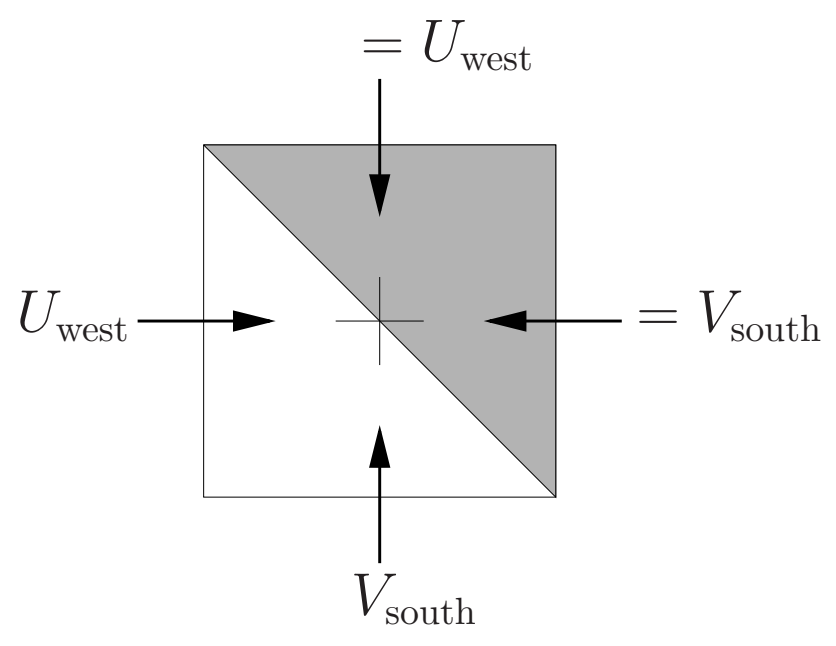

FIGURE 4: Velocity reflection in a square north-east triangular land element. A velocity equal to $U_{\text {west }}$ is directed through the northern side and one equal to $V_{\text {south }}$ through the eastern side to create a streamline along the modelled coastline. 
of each time step.

Importantly, this reflection process does not complicate the numerical procedure. In particular, Equation (4) can still be applied without modification. The only extra computations involved in modelling triangular elements entail copying some data from one array position to another at each time level. Compared with the number of operations carried out at each iteration for the finite difference formulae the additional time required for this process is insignificant.

\section{$5 \quad$ Testing the model}

Verification of results is an essential part in the development of any numerical model. In this section the theory developed thus far, and the computer code used to implement this theory, is tested using an analytic solution of Equations (1-3). Also, by computing differences between modelled results and exact analytic values, quantitative comparisons between the standard and triangulated models can be made.

The solution used to verify the model was developed in [7] for tidal propagation in a constant depth semi-infinite rectangular bay. A $60 \mathrm{~km}$ section of a $40 \mathrm{~km}$ wide bay, closed at the west end and open at the east end was considered and the response of this bay to an inward travelling semi-diurnal wave was studied. The grid spacings used in the numerical model were 
$\Delta x=\Delta y=2 \mathrm{~km}$ while a time increment of $\Delta t=30 \mathrm{~s}$ was chosen to satisfy condition (7).

In order to test the influence of triangular elements the bay was rotated anticlockwise from $\theta=0^{\circ}$ to $\theta=90^{\circ}$ at $5^{\circ}$ increments. For every angle approximate coastlines, generated with and without triangular elements, were developed and simulations were run. Rotating the bay allowed the formation of triangular elements which were not present when the bay was aligned with the gridlines. The midpoint at the eastern end of the bay was chosen as the origin of rotation, allowing perfect boundary representation for the bay to be generated when $\theta=0^{\circ}$ and $\theta=90^{\circ}$.

The model was run for a warm-up period of one tide cycle before differences were calculated. Outside the test area analytic values for surface elevations were used to avoid the introduction of further errors. Over the second wave period the root mean square (RMS) errors for both surface elevation and velocity magnitude were calculated by comparing modelled and analytic values at every $\zeta$ point inside the test area for every iteration. The RMS analytic values were also calculated over this time period allowing percentage errors to be obtained. The difference between the modelled results and the analytic solution for the various bays are shown in Figure 5.

It is clear from the graph that the relative errors are far greater for the velocities than for the water height, as is often the case in numerical tidal models. More importantly, the modelled velocities and elevations are consistently more accurate when triangular elements are used. 


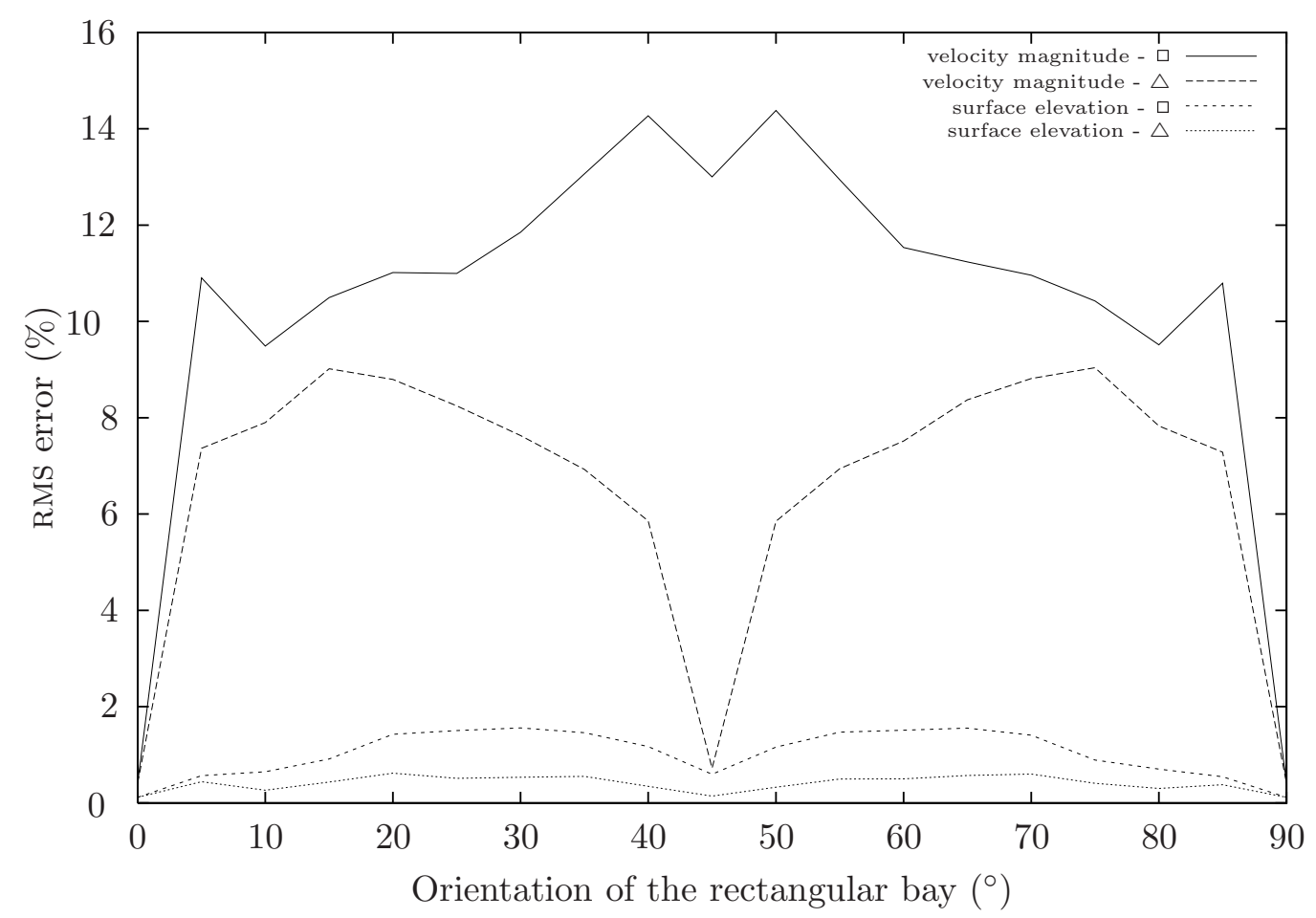

FIGURE 5: RMS errors for surface elevation and velocity magnitude with $(\triangle)$ and without $(\square)$ triangular elements. 
The improved accuracy for the velocities is particularly significant. Over the 19 orientations an average RMS error of 5.5\% is obtained using triangular elements, compared with $10.3 \%$ for the standard elements. This is a significant improvement since the only reduction in errors is due to improved accuracy of the coastline. The improvement is especially remarkable when one considers the small fraction of triangular elements introduced. For example, when the angle of rotation is $35^{\circ}$, the RMS errors for the velocity magnitude obtained using triangular and standard elements are $6.9 \%$ and $13.7 \%$ respectively. Thus a reduction of approximately $50 \%$ in RMS error is obtained by changing only $35(5.8 \%)$ of the 600 element types inside the test area.

\section{Conclusion}

A triangular coastal element for use in finite difference tidal models has been described and a method for implementing them has been presented. Without significantly increasing program run time the introduction of these elements has markedly improved the accuracy of results, as demonstrated by a simple test case. The simplicity of their modelling allows the same finite difference formulae to be used for both triangular and water elements and the reflection procedure may be implemented as a module at the end of each iteration.

In future research the present model will be modified to include forcing from meteorological terms, and non-linear advection terms will be added to 
the governing equations. The inclusion of open sea boundary conditions will also be addressed.

\section{References}

[1] A. Arakawa and V. R. Lamb. Computational design of the basic dynamical processes of the UCLA general circulation model. Methods in Computational Physics, 14:173-265, 1977. C943

[2] P. J. Bills and B. J. Noye. Verification of a three-dimensional tidal model for coastal seas. In J. Noye and C. Fletcher, editors, Computational Techniques and Applications : CTAC-83, pages 394-410. North-Holland, 1984. C938

[3] V. Casulli. Semi-implicit finite difference methods for the two-dimensional shallow water equations. Journal of Computational Physics, 86(1):56-74, 1990.

[4] R. A. Flather. Analytical and numerical studies in the theory of tides and storm surges. PhD thesis, University of Liverpool, 1972.

[5] N. S. Heaps. A two-dimensional numerical sea model. Philosophical Transactions of the Royal Society of London, A265:93-137, 1969. C938

[6] B. J. Noye and D. J. McInerney. Triangular coastal elements for finite difference tidal models. In preparation, 1999. C945 
[7] M. M. Rienecker and M. D. Teubner. A note on frictional effects in Taylor's problem. Journal of Marine Research, 38(2):183-191, 1980. C948 